\title{
Factores socio-demográficos, crecimiento y sintomatología postraumática en sobrevivientes del terremoto de Chile del 2010
}

\author{
Socio-demographic factors, posttraumatic growth and symptoms in \\ survivors of Chile earthquake 2010
}

\author{
Felipe E. García* \\ Universidad Santo Tomás, Chile \\ (Rec.: septiembre de 2016 — Acept.: diciembre de 2016)
}

\begin{abstract}
Resumen
El propósito de este estudio es evaluar la relación entre factores socio-demográficos y del evento con la sintomatología postraumática (SPT) y el crecimiento postraumático (CPT) en personas que perdieron su hogar en el terremoto de Chile del 27 de febrero de 2010. Con este fin, se seleccionaron 351 personas adultas, entre 18 y 84 años, de las cuales 63,2\% eran mujeres. Se aplicó el Inventario de Crecimiento Postraumático y la Escala de Impacto de Evento Revisada, además de un cuestionario socio-demográfico. Se evidencia en el estudio la relación significativa con la SPT o el CPT de elementos tales como sexo, edad, ingresos económicos, cambio de comuna tras el sismo y la razón de la pérdida de la vivienda Se presentan los resultados y argumenta su alcance para la promoción y prevención de salud en sobrevivientes de desastres naturales.
\end{abstract}

Palabras clave: crecimiento postraumático, sintomatología postraumática, desastre natural, desplazamiento, terremoto, tsunami.

\begin{abstract}
The purpose of this study is to evaluate the relationship that socio-demographic and event factors share with posttraumatic symptomatology (PTSD) and posttraumatic growth (PTG) in people who lost their home in the earthquake that hit Chile on february 27th, 2010. To this end, 351 adults from 18 to 84 years old were selected, of whom $63.2 \%$ were women. For this study, the Posttraumatic Growth Inventory (PTGI) and the revised Impact of Event Scale (IES) were applied, in addition to a socio-demographic questionnaire. This study shows that elements such as gender, age, economic income, change of location after the earthquake, and the reason behind the loss of housing bear a significant relation with PTSD or PTG. Results are presented, and their consequences for health promotion and prevention in survivors of natural disasters are discussed.
\end{abstract}

Keywords: posttraumatic growth, posttraumatic symptoms, natural disaster, displacement, earthquake, tsunami. 


\section{Introducción}

Los eventos traumáticos son sucesos de gran intensidad, imprevisibles e infrecuentes que pueden llegar a abrumar los recursos de las personas (Gaborit, 2006). Entre las consecuencias psicológicas de estos eventos, el trastorno por estrés postraumático o su sintomatología asociada (SPT) ha sido la más estudiada, determinándose a la fecha distintos factores de riesgo en el caso de desastres naturales, entre los que destacan el grado de exposición al evento adverso y la pérdida del hogar (García, 2011).

Dentro de los eventos traumáticos que puede vivir una persona, los terremotos poseen características particulares que explican por qué sus efectos psicológicos son muchas veces mayores que el de otros tipos de desastres naturales (McCaughey, Hoffman \& Llewellyn, 1994). Una de esas características es su carácter impredecible, que lo distingue de inundaciones, huracanes y erupciones volcánicas. Esta imprevisibilidad genera un fuerte sentimiento de indefensión psicológica, que aumenta la ansiedad y la incertidumbre cognitiva sobre qué hacer cuando suceda un desastre de estas características. Además, poseen un poder destructivo sin igual; no hay nada que se pueda hacer para controlar la energía liberada por un terremoto. La potencia con la que golpea puede cubrir un área extensa y generar destrucción masiva aún en lugares distantes al epicentro. Por otro lado, las réplicas, que pueden ser muchas y durar bastante tiempo, generan efectos psicológicos importantes, ya que crean las condiciones para la reexperimentación del trauma inicial, lo que tiende a elevar más aún el estado de hipervigilancia de las víctimas. Esta re-experimentación dificulta la recuperación de las personas y las comunidades (Gaborit, 2006). Consecuentemente, los terremotos alteran súbita y radicalmente por un tiempo también impredecible, las rutinas individuales, planes, proyectos y estilos de vida, que requieren una fuerte capacidad de adaptación en las personas (Gaborit, 2006).

La madrugada del día 27 de febrero de 2010, la región centro sur de Chile fue devastada por un terremoto magnitud 8.8 Mw, con epicentro en la localidad de Cobquecura (Vargas et al., 2011). Este sismo (en adelante, 27/F) es considerado como la peor tragedia natural de los últimos 50 años en Chile, después del cataclismo de 1960 en Valdivia y el sexto sismo de mayor magnitud registrado en el mundo desde inicios del siglo XX (United States Geological Survey, 2017). Larrañaga y Herrera (2010), usando la escala autoadministrada de Davidson, encontraron un $12 \%$ de TEPT tres meses después del 27/F en población adulta afectada, obteniendo un porcentaje más alto los habitantes de la Región del Bío-Bío (23.9\%). Leiva y Araneda (2013), usando la escala de Davidson, obtuvieron una prevalencia del $22 \%$ en la muestra total y un $33 \%$ en quienes sufrieron daños en sus viviendas.

La pérdida del hogar o el desplazamiento, sumado al sexo femenino (Baddam, Russel \& Swamidhas,
2007; Batniji, Van Ommeren \& Saraceno, 2006; John, Russell \& Russell, 2007; Tang, 2006; Tural et al., 2001; Xu \& Song, 2011), constituyen un factor de riesgo para la presencia de TEPT y otros problemas de salud mental en población afectada por un desastre natural. La pérdida del hogar y la relocalización más o menos permanente de las personas en nuevos contextos, produce que las consecuencias se prolonguen en el tiempo, complejizando la visión de los desastres como eventos agudos (Cova \& Rincón, 2010). Por tanto, es esperable un efecto severo de estas condiciones sobre el bienestar de las personas.

Larrañaga y Herrera (2010) estiman que un $8.8 \%$ de la población en las seis regiones afectadas por el terremoto del $27 / \mathrm{F}$ y, un $17.8 \%$ de los habitantes de la región del Bío-Bío, sufrieron la destrucción parcial o total de sus viviendas. Leiva y Araneda (2013) señalan que estas personas se muestran más tristes, tienen menos cuidado por su salud, sienten incomodidad con sus reacciones posteriores al evento, y que estas reacciones interfieren en sus actividades cotidianas, al igual que les resulta difícil resolver problemas que antes solucionaban sin ayuda. Jóvenes desplazados por el terremoto de Loma Prieta en EE.UU, percibían el hecho de vivir en campamentos como particularmente frustrante y desmoralizante (Nolen-Hoeksema \& Morrow, 1991). El sufrimiento producido por el sismo se mantiene por la no resolución del tema habitacional. Leiva y Araneda (2013) observan mayores niveles de TEPT en quienes sufrieron graves daños en sus viviendas, atribuible al estar continuamente expuestos al contexto de destrucción que significó el evento.

Sin embargo, no todas las personas expuestas a un trauma responden de esta manera. Al parecer, algunas cuentan con recursos que les permiten afrontar dichos eventos, incluso percibiendo cambios positivos en sus vidas a partir de estas vivencias. El interés en el estudio de esta percepción es relativamente reciente. En 1996, Tedeschi y Calhoun proponen el constructo de crecimiento postraumático para aludir a los cambios positivos que un individuo experimenta como resultado del proceso de lucha ante un trauma. A partir de ese momento, se han efectuado diversas investigaciones que intentan comprender los factores que determinan este crecimiento.

El enfocarse en la experiencia de aprendizaje y crecimiento no niega el dolor ni la miseria en el ser humano. Por el contrario, el crecimiento postraumático coexiste con el sufrimiento. Parte de las personas que experimentan dicho crecimiento continúan experimentando emociones negativas resultantes de la experiencia traumática (tristeza, ira, culpa, irritabilidad). Incluso, como proponen Calhoun y Tedeschi (1998), es posible que para experimentar dicho crecimiento sea necesaria la coexistencia de emociones positivas y negativas. En otras palabras, es probable que el sufrimiento producido por determinadas experiencias desencadene un aprendizaje. En muchos casos, sin la presencia de estas emociones negativas, al menos en su inicio, el CPT no se dará. Esto explicaría por qué en algunos 
estudios el TEPT y el CPT se presentan juntos (Hafstad, Gil-Rivas, Kilmer \& Raeder, 2010; Kilmer \& Gil-Rivas, 2010; McCaslin et al., 2009) o que se haya informado de una relación positiva entre severidad subjetiva y CPT (García, Jaramillo, Martínez, Valenzuela \& Cova, 2014) al igual entre severidad subjetiva y TEPT (Boals \& Schuettler, 2009; Morris, Shakespeare-Finch, Rieck \& Newberry, 2005). Calhoun y Tedeschi (1998) sugieren que debido a la ruptura de los supuestos fundamentales que han dado significado a la vida, el mismo trauma pone en marcha tanto procesos que conducen al TEPT como al CPT.

La evidencia sobre el efecto del CPT en el ajuste psicológico mediato y posterior ha sido poco consistente. Estudios más actuales, sin embargo, se han aproximado a una respuesta a este importante asunto; por ejemplo, una revisión de Tennen y Afleck (2005) concluyó que el CPT se asocia en 14 estudios de 20, a un mejor ajuste emocional. En un metaanálisis realizado por Helgeson, Reynolds y Tomich (2006), se encontró que un mayor CPT se asociaba con una menor depresión y un mayor bienestar psicológico. Estos mismos resultados se encuentran en una revisión de estudios longitudinales realizado por Zoellner y Maercker (2006a), el cual muestra que el CPT predice menor depresión y TEPT a largo plazo; los autores señalan que una posible explicación de estos resultados es la asociación entre CPT y estrategias más saludables y apropiadas para afrontar acontecimientos traumáticos.

Vázquez, Castilla y Hervás (2009) destacan en su revisión que los estudios longitudinales muestran que el CPT predice futuras disminuciones del malestar en casos de exposición a traumas severos. Lo cual indicaría que, si bien disminuir el malestar psicológico no necesariamente promueve el crecimiento, experimentar crecimiento sí promueve el ajuste postraumático y alivia dicho malestar.

El objeto de este estudio es evaluar la relación entre diversas variables socio-demográficas y del evento con el CPT y la SPT en personas expuestas a un desastre natural que les implicó la pérdida de su hogar. Se persigue identificar las variables sociodemográficos que inciden en el desarrollo de CPT y SPT y contribuir a la promoción de respuestas más adaptativas o en la prevención de respuestas desadaptativas tras un desastre natural.

\section{Método}

El presente estudio utiliza un diseño de investigación descriptivo y correlacional; se trata de un estudio no experimental ex-post-facto. Los datos fueron tomados en un solo corte temporal, por lo que corresponde a un estudio transversal.

\section{Participantes}

Se evaluó a 351 personas, 222 mujeres (63.2\%) y 129 hombres (36.8\%), que perdieron su hogar debido al terremoto y tsunami que afectó a la Región del Bío-Bío, Chile, el 27/F de 2010, en edades comprendidas entre los 18 y los 84 años $(M=40.4$, $D E=15.29$ ).

\section{Instrumentos}

Para evaluar el CPT, se utilizó el Posttraumatic Growth Inventory (PTGI) de Tedeschi y Calhoum (1996), validada en Chile por García, Cova y Melipillán (2013). Consta de 21 ítems que se responden en una escala Likert de 6 grados, de 0 ("ningún cambio") a 5 puntos ("un cambio muy importante"). El puntaje total obtenido varía desde 0 a 105. Sus creadores obtuvieron una consistencia interna de $\alpha=0.90$ y una confiabilidad test-retest de $r=0.71$, además de mostrar validez discriminante en relación a otros constructos y validez de constructo al evaluar específicamente los cambios positivos obtenidos a partir de un acontecimiento traumático y no cualquier cambio. En su adaptación chilena se obtuvo un $\alpha=0.95$.

Para medir la sintomatología postraumática, se utilizó la Escala de Impacto de Evento-Revisada (Weiss \& Marmar, 1997), validada en Chile por Caamaño et al. (2011). Consta de 22 ítems que se responden en una escala Likert desde nada $=0$ a extremadamente $=4$. El puntaje máximo es de 88 puntos. En el estudio de Caamaño et al. (2011), informan un alfa de Cronbach de 0.98 .

Se elaboró además un cuestionario sociodemográfico y del evento en donde se consultó sobre las siguientes variables: edad, género, nivel socio-económico, ciudad en la que estaba cuando ocurrió el terremoto, ciudad de residencia actual y causa del daño de su vivienda.

\section{Procedimiento}

Se reclutaron 55 encuestadores voluntarios entre psicólogos titulados y estudiantes de psicología, quienes fueron previamente capacitados con el objeto de obtener uniformidad. Para acceder a los participantes se empleó un muestreo por accesibilidad a través del método bola de nieve. Los encuestadores aplicaron los instrumentos tanto a personas conocidas como personas referidas por aquellos que cumplían con los criterios de inclusión y exclusión. También se dirigieron a caletas de pescadores y campamentos de damnificados de la provincia de Concepción, previo contacto con dirigentes vecinales. La participación fue de carácter voluntario, las identidades de los participantes se manejaron con discreción y se garantizó la confidencialidad de los datos entregados en las distintas encuestas que sólo fueron analizados en su conjunto. Información contenida en el protocolo de consentimiento que se les pedía firmar previo a la aplicación de las encuestas.

El proyecto fue revisado y aprobado por el Comité del Doctorado en Psicología de la Universidad de Concepción, siguiendo los principios éticos habituales para la investigación psicológica de acuerdo a la American Psychological Association (2010). 


\section{Análisis de datos}

Se realizó un análisis exploratorio/descriptivo a través de estadísticos de tendencia central, variabilidad y de análisis de gráficos. A continuación, se realizaron análisis de comparación de grupos para contrastar las distintas características sociodemográficas y del evento con las variables dependientes del estudio, es decir, SPT y CPT. Para estos análisis de utilizó el programa estadístico SPSS v18.

\section{Resultados}

Como muestra la tabla 1 , se realizó un análisis descriptivo previo a la adecuación de los modelos de medida para evaluar puntajes mínimos, máximo, media y desviación estándar de los instrumentos utilizados en este estudio. Igualmente, se analizó asimetría y curtosis así como la consistencia interna.

Tabla 1. Estadísticos descriptivos y confiabilidad ( $\alpha$ de Cronbach) de los instrumentos de estudio $(\mathrm{N}=351)$

\begin{tabular}{lccccccc}
\hline & Mínimo & Máximo & $\mathrm{M}$ & $\mathrm{DE}$ & Asimetría & Curtosis & $\alpha$ \\
\hline SPT & 0 & 36 & 10.76 & 9.60 & 0.73 & -0.55 & 0.94 \\
CPT & 0 & 45 & 29.22 & 11.19 & -0.73 & -0.26 & 0.92
\end{tabular}

$\mathrm{SPT}=$ Sintomatología postraumática; CPT=Crecimiento postraumático

Como se observa, la asimetría y curtosis son indicadores de que los instrumentos utilizados tienen una distribución normal, ya que los valores se ubican en el rango de +1 a -1 . Así mismo, las consistencias internas son adecuadas para ambos instrumentos. En relación al CPT y a la SPT, se realizaron comparaciones de grupo para evaluar si existían diferencias significativas entre las distintas características socio-demográficas. Para ello se utilizó la $t$ de student para las variables sexo y cambio de comuna, y el ANOVA de un factor, con contraste post-hoc HSD de Tukey, para las demás. Los resultados se exponen a continuación.

En relación al sexo y el CPT, las mujeres alcanzaron un promedio de 69.39 ( $D E=24.61)$; en cambio, los hombres alcanzaron un promedio de 62.74 $(D E=25.59)$. Los resultados estadísticos del $t d e$ student para muestras independientes fueron $t$ $(349)=2.425 ; \quad p<0.016$. Confirmándose así, que existen diferencias significativas en sexo, obteniendo las mujeres mayores niveles de CPT que los hombres.

En relación a la SPT, las mujeres alcanzaron un promedio de 29.99 ( $D E=22.33$ ) y los hombres, de 20.95 ( $D E=19.34)$. Los resultados estadísticos del $t$ de student para muestras independientes fueron $t$ $(349)=3.837 ; p<0.001$. En consecuencia, se presentan diferencias significativas en sexo, obteniendo las mujeres mayores niveles de SPT que los hombres.

En relación al cambio de comuna y el CPT, los resultados de la prueba $t$ de student muestran que no existen diferencias significativas entre ambos grupos.

En relación al SPT, quienes tuvieron que cambiarse de comuna de residencia tras el $27 / F$ obtuvieron un promedio de 27.68 ( $D E=21.82$ ); en cambio, quienes permanecieron en su comuna de residencia obtuvieron un promedio de 19.93 ( $D E=19.83)$. Los resultados estadísticos del $t$ de student para muestras independientes fueron $t(349)=2.270$; $p<0.024$, indicando que existen diferencias significativas en ambos grupos, obteniendo aquellos que tuvieron que cambiarse de comuna niveles de SPT más altos que quienes permanecieron viviendo en la misma comuna.

En relación al rango de edad y el CPT, los resultados de la prueba ANOVA de un factor muestran que no existen diferencias significativas entre los grupos.

Al comparar por niveles de edad en relación a la SPT, los resultados de la prueba ANOVA de un factor correspondieron a $F(2,348)=4.775 ; p<0.009$. Resultados que señalan la existencia de diferencias estadísticamente significativas en los niveles de SPT entre el nivel de edad de 18-39 años $(M=23.16$; $\mathrm{DE}=21.62)$ y los niveles de edad de 40-59 años $(M=30.01 ; D E=21.95)$ y de $60-84$ años $(M=30.93$; $D E=19.05)$, siendo más bajo el nivel de SPT en el grupo más joven. No hubo diferencias significativas entre los grupos de adultez media y mayor.

Al comparar por ingreso per cápita/hogar en relación al CPT, los resultados de la prueba ANOVA de un factor correspondieron a $F(2,348)=4.693 ; p<0.01$. Con lo cual, existen diferencias estadísticamente significativas en los niveles de CPT entre los niveles de ingreso medio $(M=60.29 ; D E=27.14)$ con los niveles alto $(M=73.54 ; D E=21.28)$ y bajo $(M=68.60$; $D E=24.09$ ), obteniendo menores niveles de CPT aquellos con nivel de ingresos medio. No hubo diferencias significativas entre los niveles bajo y alto.

Al comparar por ingreso per cápita/hogar en relación a la SPT, los resultados de la prueba ANOVA de un factor correspondieron a $F(2,348)=6.328 ; p<0.002$. Estos resultados indican que existen diferencias estadísticamente significativas en los niveles de SPT entre los niveles de ingreso bajo $(M=29.24$; $D E=22.11)$ y alto $(M=16.29 ; D E=18.01)$, obteniendo mayores niveles de SPT aquellos con más bajo nivel de ingresos. No hubo diferencias significativas con el nivel de ingresos medio. 
En relación a la razón de la pérdida de la vivienda y el CPT, los resultados de la prueba ANOVA de un factor muestran que no existen diferencias significativas entre los grupos.

En cuanto a la SPT, los resultados de la prueba ANOVA de un factor correspondieron a $F$ $(2,335)=3.573 ; p<0.029$. Señalando así que existen diferencias estadísticamente significativas en los niveles de SPT entre las personas que perdieron su hogar debido al tsunami $(M=29.78 ; D E=20.66)$ y quienes su edificio fue declarado inhabitable $(M=20.27 ; D E=19.10)$, siendo más alto en quienes fueron afectados por el tsunami. No se observan diferencias significativas con quienes perdieron su hogar por daños

en la estructura de su casa. En la tabla 2 se resumen los contrastes realizados.

Tabla 2. Comparaciones de grupo en SPT y CPT de acuerdo a características socio-demográficas y del evento $(\mathrm{N}=351)$

\begin{tabular}{|c|c|c|c|c|}
\hline & & & CPT & SPT \\
\hline & & $n(\%)$ & $M(D E) \mathrm{CPT}$ & $M(D E) \mathrm{SPT}$ \\
\hline \multirow[t]{4}{*}{ Edad } & 18-39 años & $177(50.4)$ & $65.25(24.68)$ & $23.16(21.62)$ \\
\hline & 40-59 años & 133 (37.9) & $68.02(25.97)$ & 30.01 (21.95) \\
\hline & 60-84 años & $41(11.7)$ & $70.80(22.37)$ & $30.93(19.05)$ \\
\hline & & & $F=1.022$ & $F=4.775^{\star *}$ \\
\hline \multirow[t]{3}{*}{ Sexo } & Mujer & $222(63.2)$ & $69.39(24.61)$ & $29.99(22.33)$ \\
\hline & Hombre & $129(36.8)$ & $62.74(25.59)$ & 20.95 (19.34) \\
\hline & & & $t=2.425^{\star}$ & $t=3.837^{* * *}$ \\
\hline \multirow[t]{4}{*}{ Ingreso per capita/hogar } & Nivel bajo & 237 (67.5) & $68.60(24.09)$ & $29.24(22.11)$ \\
\hline & Nivel medio & $86(24.5)$ & $60.29(27.14)$ & $22.94(20.26)$ \\
\hline & Nivel alto & $28(8)$ & $73.54(21.28)$ & $16.29(18.01)$ \\
\hline & & & $F=4.693^{* *}$ & $F=6.328^{* *}$ \\
\hline \multirow{3}{*}{$\begin{array}{l}\text { Cambio de comuna tras } \\
\text { sismo }\end{array}$} & No cambió & 305 (86.9) & $67.85(24.47)$ & $19.93(19.83)$ \\
\hline & Sí cambio & $46(13.1)$ & $60.93(27.31)$ & $27.68(21.82)$ \\
\hline & & & $t=1.760$ & $t=2.270^{*}$ \\
\hline \multirow{4}{*}{$\begin{array}{l}\text { Razón pérdida de } \\
\text { vivienda }\end{array}$} & Casa dañada & $188(53.6)$ & $65.51(24.75)$ & $27.81(22.76)$ \\
\hline & Tsunami & $95(27.1)$ & $71.30(21.41)$ & $29.78(20.66)$ \\
\hline & Edificio inhabitable & $55(15.7)$ & $70.58(25.11)$ & $20.27(19.10)$ \\
\hline & & & $F=2.248$ & $F=3.573^{*}$ \\
\hline
\end{tabular}




\section{Discusión}

La presente investigación perseguía identificar las características sociodemográficas y del evento que se relacionaban con la presencia de SPT y CPT en personas que perdieron su hogar debido al terremoto y/o tsunami de Chile del 27/F 2010.

En relación al sexo, los niveles de CPT observados fueron más altos en mujeres que en hombres. La mayoría de los estudios dan cuenta que las mujeres presentan mayores niveles de CPT que los hombres (ver metanálisis de Helgeson et al., 2006 y de Vishnevsky, Cann, Calhoun, Tedeschi \& Demakis, 2010). Una posible explicación de estas diferencias tiene que ver con la mayor percepción de amenaza por parte de las mujeres (Olff, Langeland, Draijer \& Gersons, 2007). De acuerdo con el modelo de CPT de Calhoun y Tedeschi (2006), una mayor percepción de amenaza puede llevar a un mayor impacto sobre creencias fundamentales acerca del mundo en un individuo, lo que sienta las bases a informar mayores niveles de CPT en el futuro. Un posible mediador entre el género y el CPT encontrado en el meta-análisis de Vishnevsky et al. (2010), es la tendencia de las mujeres a tener más pensamientos rumiativos que los hombres (Treynor, Gonzalez \& Nolen-Hoeksema, 2003). La tendencia a rumiar en temas constructivos, tales como una mayor conciencia de las fortalezas personales o una apreciación de la importancia de las conexiones sociales, ha sido sugerida como un mecanismo que conduce a mayores niveles de CPT (Janoff-Bulman, 2006; Tedeschi \& Calhoun, 2004); por lo tanto, si las mujeres presentan más rumiación deliberada, pueden también reconocer más beneficios a través de sus esfuerzos para hacer frente a un suceso traumático y reportar mayores niveles de CPT (Vishnevsky et al., 2010).

En relación a la SPT, las mujeres muestran niveles significativamente más altos que los hombres. Las diferencias de género así como la relación con acontecimientos potencialmente traumáticos son las bases del meta-análisis realizado por Tolin y Foa (2006), cuyos resultados muestran que mientras los hombres tienen más probabilidades de experimentar un evento traumático, las mujeres ante el mismo estresor son más propensas a cumplir con los criterios para el diagnóstico de TEPT. Con estos hallazgos, Tolin y Foa (2006) sugieren que el aumento de riesgo de las mujeres para el TEPT no se debe necesaria o exclusivamente a mayores tasas de exposición a ciertos tipos de trauma, sino a factores como las diferencias en el procesamiento cognitivo o afectivo de eventos traumáticos. Al respecto, Caro y Cova (2012) en un estudio con estudiantes expuestos al terremoto de Chile del año 2010, encontraron diferencias significativas de género tanto en SPT como en rumiación depresiva, con mayores niveles en mujeres. Reafirmándose así la posibilidad de que diferencias en el procesamiento cognitivo estén implicadas en los mayores niveles de SPT en las mujeres.

En relación a la edad, no se encontraron diferencias significativas en CPT. Diversos estudios son poco concluyentes en establecer la naturaleza de esta relación (Helgeson et al., 2006). Por ejemplo, Schroevers, Helgeson, Sanderman y Ranchor (2010) encontraron una relación negativa de la edad con CPT en mujeres sobrevivientes de cáncer al igual que García et al. (2016) en pobladores expuestos a violencia social; Grubaugh y Resick (2007) encontraron una relación positiva en mujeres víctimas de abuso físico y sexual; Cieslak et al. (2009) no encontraron ninguna relación en pacientes con VIH expuestos a un desastre natural. Esto indicaría que la edad jugaría un rol diferente dependiendo del tipo de evento que se trate y de la severidad percibida.

Sí se observaron diferencias significativas entre edad y SPT, siendo más bajos los niveles de SPT en el grupo etario correspondiente a adulto joven (18 a 39 años), en comparación al adulto medio (40-59 años) y mayor (>60 años). En el meta-análisis de Orth y Wieland (2006), el promedio de edad no aporta de forma significativa la predicción de TEPT. En un estudio sobre los efectos de una tragedia aérea, los menores de 35 años desarrollaban más sintomatología postraumática que los mayores de 35 años (Gregg et al., 1995), los más jóvenes también desarrollan más TEPT que los de mayor edad en víctimas del terrorismo (Trautman et al., 2002). Una posible explicación a los menores niveles de SPT en el presente estudio en el grupo de menor edad se basa en el efecto de perder el hogar, el cual es más alto en personas establecidas en un lugar físico y posiblemente habiendo formado familia, normalmente personas de mayor edad, que en quienes se encuentran estudiando o en sus primeros años laborales.

La evidencia disponible no muestra relaciones entre nivel socio-económico (NSE) y CPT en población general (Helgeson et al., 2006). Sin embargo, en el presente estudio las personas de NSE medio presentaron niveles de CPT significativamente más bajos que las personas de mayores o de menores ingresos; en cambio, entre estos últimos, no se observaron diferencias. Una posible explicación puede ser que el efecto de la pérdida de las viviendas y de otras consecuencias del terremoto en sus vidas cotidianas son mayores en los niveles más bajos (Leiva \& Araneda, 2013) y, por lo tanto, es mayor la necesidad de encontrar beneficios ante esa experiencia (Tomich \& Helgeson, 2004).

Sin embargo, no explica por qué el CPT en el NSE bajo fue mayor que el grupo de NSE medio y no que el NSE alto. Algunos estudios han observado mayores niveles de CPT en población de ingresos altos que bajo (Updegraff, Taylor, Kemeny \& Wyatt, 2002), pero los procesos implicados no son claros; resulta razonable pensar que esta relación podría estar confundida con otras variables psicosociales que tuvieran una relación más clara con el CPT (Vázquez et al., 2009). Sí se observaron diferencias entre el nivel socio-económico bajo y el alto en los niveles de SPT, lo que resulta esperable dada la mayor vulnerabilidad ante los impactos del terremoto de las personas de menores ingresos (Bisson, 2007; 
Galea, Nandi \& Vlahov, 2005; Leiva \& Araneda, 2013).

El cambio de comuna después del terremoto supone un estresor adicional en personas que han perdido su hogar, pues el desplazamiento se produce ya no solo de la vivienda sino también desde la comunidad en el que habitualmente las personas desarrollaban sus actividades (Fullilove, 1996). Al respecto, no se encontraron diferencias significativas entre los grupos en relación al CPT. En cuanto a la SPT, el grupo que se cambió de comuna mostró niveles más altos que aquellos que permanecieron en ella después de haber perdido su hogar. Las recolocaciones, incluso cuando son en zonas mejor provistas, no disminuyen la prevalencia del TEPT (Najarian, Goenjian, Pelcovitz, Mandel \& Najarian, 2001). Kilic y Ulusoy (2003) observaron que los sobrevivientes que se desplazaron a barrios más seguros tras un sismo, presentaban mayores niveles de TEPT que quienes habían permanecido en su comunidad. Estos hallazgos, apoyan la idea de que la relocalización más o menos permanente de las personas en nuevos contextos tras un desastre favorece la prolongación en el tiempo de las consecuencias negativas (Cova \& Rincón, 2010).

Las personas perdieron sus viviendas por diferentes razones: daños estructurales en el hogar, edificio declarado inhabitable o destrucción por tsunami. No se observaron diferencias significativas en relación al CPT en función de la causa de la pérdida de la vivienda. En cambio, sí se observaron diferencias en relación a la SPT. Las personas que perdieron su hogar por el tsunami presentaban niveles más altos que quienes su edificio fue declarado inhabitable. Probablemente, estas diferencias son atribuibles al tsunami. En el desastre del $27 / F$, el tsunami fue particularmente devastador y existe evidencia de que las personas que fueron expuestas a éste presentaron más consecuencias negativas que quienes se vieron expuestos solo al terremoto (Cova et al., 2013).

Los datos del presente estudio muestran una correlación bivariada positiva y significativa pero leve, entre CPT y SPT $(r=0.24 ; p<0,001)$. Esta relación coincide con lo observado en diversos estudios que muestran una relación lineal positiva entre CPT y SPT o el TEPT (Hafstad et al., 2010; Kilmer \& Gil-Rivas, 2010; McCaslin et al., 2009), en especial en poblaciones afectadas por un desastre natural (Shakespeare-Finch \& Lurie-Beck, 2014). Esto se explicaría con lo planteado por Calhoun y Tedeschi (1998), quienes sugieren que el trauma pone en marcha tanto procesos que conducen al TEPT como al CPT, ambos relacionados al quiebre de las creencias básicas acerca del mundo y a su necesidad de reconstrucción. Calhoun y Tedeschi (1998) plantean que es justamente por el sufrimiento que logramos aprender algo de determinadas experiencias. Así como el malestar asociado a un evento potencialmente traumático tiende a ser más intenso en los primeros momentos, con el paso del tiempo el carácter constructivo del CPT va aumentando a medida que el afrontamiento al estrés se hace más exitoso (Zoellner \& Maercker, 2006b). Estudios longitudinales han mostrado que el CPT es una respuesta al SPT inicial y no al revés, encontrando que el malestar poco después de un evento predice el CPT cinco años después (Dekel, Ein-Dor \& Solomon, 2012).

Este estudio presenta varias limitaciones. La toma de datos se realizó dos años después del terremoto, por lo que sus resultados podrían ser diferentes a los obtenidos inmediatamente después del evento. No obstante, los sesgos de recuerdo se minimizan, ya que se pidió a los participantes que respondieran como se encontraban en el último tiempo en su SPT y CPT y no en los momentos cercanos al evento. Igualmente, el muestreo por accesibilidad utilizado pudo generar sesgo de selección, es decir, los evaluadores pudieron influir en que un sujeto con determinadas características se incluyera o no en el estudio, limitando la generalización de estos resultados.

Los resultados de este estudio abren espacio para futuras investigaciones que continúen explorando las respuestas psicológicas de los individuos frente a los eventos traumáticos no sólo desde la patología, sino también desde la perspectiva de la psicología positiva. El enorme número de personas afectadas por desastres naturales, acentúa la necesidad de investigar y socializar a las comunidades sobre las medidas que se deben tomar para hacer frente a estos sucesos, con el fin de reducir el impacto negativo en las vidas de las personas y a su vez orientar intervenciones psicosociales destinadas a promover respuestas más adaptativas tras un desastre.

En esa línea, el presente estudio identifica factores relacionados con el aumento del SPT. Las intervenciones psicosociales iníciales deberían orientarse hacia estas personas, principalmente de sexo femenino, adultos medios o mayores, de nivel socio-económico bajo, afectados por el tsunami y que en el desplazamiento hacia una nueva vivienda tuvieron que desarraigarse de su comunidad. Así, considerando el efecto que el cambio de comuna podría producir en la salud mental tras un desastre, las políticas públicas dirigidas a cubrir necesidades habitacionales en quienes perdieron su hogar, deben considerar la necesidad de mantener a estas personas conectadas con su comunidad de origen.

Respecto al CPT, igualmente se concluye que las mujeres y las personas de nivel socio-económico medio son quienes más crecen. González (2004) señala que la labor del psicólogo debe ir por el camino de reorientar a las personas para que encuentren la manera de aprender y crecer con su experiencia traumática. Creemos que los resultados de este estudio contribuyen a dar un paso hacia ese fin.

\section{Referencias}

American Psychological Association. (2010). Ethical principles of psychologists and code of conduct. Washington DC: Author. 
Baddam, P., Russel, S, \& Swamidhas, P. (2007). The prevalence of posttraumatic stress disorder among children and adolescents affected by tsunami disaster in Tamil Nadu. Disaster Management \& Response, 5, 3-7. doi: 10.1016/j.dmr.2006.11.001

Batniji, R., Van Ommeren, M. \& Saraceno, B. (2006). Mental and social health in disasters: relating qualitative social science research and the Sphere standard. Social Science \& Medicine, 62, 1853-1864.

10.1016/j.socscimed.2005.08.050

Bisson, J. (2007). Post-traumatic stress disorder. British Medical Journal, 334(7597), 789-793. doi:10.1136/bmj.39162.538553.80

Boals, A. \& Schuettler, D. (2009). PTSD symptoms in response to traumatic and non-traumatic events: The role of respondent perception and $\mathrm{A} 2$ criterion. Journal of Anxiety Disorders, 23, 458462. doi: 10.1016/j.janxdis.2008.09.003

Caamaño, L., Fuentes, D., González, L., Melipillán, R., Sepúlveda, M. \& Valenzuela, E. (2011). Adaptación y validación de la versión chilena de la escala de impacto de evento-revisada (EIE-R). Revista Médica de Chile, 139(9), 1163-1168. doi:10.4067/S0034-98872011000900008

Calhoun, L. \& Tedeschi, R. (1998). Beyond recovery from trauma: Implications for clinical practice and research. Journal of Social Issues, 54(2), 357371. doi:10.1111/j.1540-4560.1998.tb01223.x

Calhoun, L. \& Tedeschi, R. (2006). The foundations of posttraumatic growth: An expanded framework. En L. Calhoun \& R. Tedeschi (Eds.), Handbook of posttraumatic growth: Research and practice (pp. 3-23). New Jersey: Lawrence Erlbaum Associates.

Caro, P. \& Cova, F. (2012). Relación entre estrés postraumático, afrontamiento represivo $y$ rumiación en estudiantes universitarios chilenos. Ciencia y Enfermería, 18(3), 121-130. doi:10.4067/S0717-95532012000300012

Cieslak, S., Benight, C., Schmidt, N., Luszczynska, A., Curtin, E., Clark, R. \& Kissinger, P. (2009). Predicting posttraumatic growth among Hurricane Katrina survivors living with HIV: the role of self-efficacy, social support, and PTSD. Anxiety, Stress \& Coping, 22(4), 449-463. doi:10.1080/10615800802403815

Cova, F. \& Rincón, P. (2010). El terremoto y tsunami del 27-f y sus efectos en la salud mental. Terapia Psicológica, 28(2), 179-185. doi:10.4067/S071848082010000200006

Cova, F., Valdivia, M., Rincón, P., Haquin, C., Sanhueza, F., Melipillán, R., ... \& Alarcón, G. (2013). Estrés postraumático en población infantojuvenil post 27F. Revista Chilena de Pediatría, 84, 32-41. doi:10.4067/S0370-41062013000100004

Dekel, S., Ein-Dor, T., \& Solomon, Z. (2012). Posttraumatic growth and posttraumatic distress: A longitudinal study. Psychological Trauma: Theory, Research, Practice, and Policy, 4, 94-101. doi:10.1037/a0021865
Fullilove, M. (1996). Psychiatric implications of displacement: Contributions from the psychology of place. The American Journal of Psychiatry, 153(12), 1516-1523. doi:10.1176/ajp.153.12.1516

Gaborit, M. (2006). Desastres y trauma psicológico. Pensamiento Psicológico, 2(7), 15-39. Recuperado de http://www.redalyc.org/pdf/801/80120703.pdf

Galea, S., Nandi, A. \& Vlahov, D. (2005). The epidemiology of post-traumatic stress disorder after disasters. Epidemiologic Reviews, 27, 78-91. doi:10.1093/epirev/mxi003

García, F. (2011). Prevention of psychopatholgical consequences in survivor of tsunamis. En $\mathrm{M}$. Mokhtari (Ed.), Tsunami: A Growing Disaster (pp. 211-232). Rijeka: InTech.

García, F., Cova, F. \& Melipillán, R. (2013). Propiedades psicométricas del Inventario de Crecimiento Postraumático en población chilena afectada por un desastre natural. Revista Mexicana de Psicología, 30(2), 145-153. Recuperado de http://www.redalyc.org/pdf/2430/243033029007. pdf

García, F., Capponi, C., Hinrichs, F., Lillo, F., Rodríguez, C., \& Sánchez, J. (2016). Violencia policial y afrontamiento: Crecimiento postraumático en pobladores de una comunidad aislada del sur de Chile. Universitas Psychologica, 15(4), 1-12. doi: 10.11144/Javeriana.upsy15-4.vpac

García, F., Jaramillo, C., Martínez, A.M., Valenzuela, I. \& Cova, F. (2014). Respuestas psicológicas ante un desastre natural: Estrés y crecimiento postraumático. Liberabit, 20, 121-130. Recuperado de http://www.scielo.org.pe/pdf/liber/v20n1/a11v20 n1.pdf

González, C. (2004). La Psicología Positiva: Un cambio en nuestro enfoque patológico clásico. Liberabit, 10 , 82-88. Recuperado de http://www.redalyc.org/pdf/686/68601009.pdf

Gregg, W., Medley, I., Fowler-Dixon, R., Curran, P., Loughrey G., Bell, P., ... \& Harrison, G. (1995). Psychological consequences of the Kegworth air disaster. British Journal of Psychiatry, 167(6), 812-817. doi:10.1192/bjp.167.6.812

Grubaugh, A. \& Resick, P. (2007). Posttraumatic growth in treatment-seeking female assault victims. Psychiatric Quarterly, 78(2), 145-155. doi:10.1007/s11126-006-9034-7

Hafstad, G., Gil-Rivas, V., Kilmer, R. \& Raeder, S. (2010). Posttraumatic growth among Norwegian children and adolescents following a natural disaster. American Journal of Orthopsychiatry, 80(2), 248257. doi:10.1111/j.1939-0025.2010.01028.x

Helgeson, V., Reynolds, K. \& Tomich, P. (2006). A metaanalytic review of benefit finding and growth. Journal of Consulting and Clinical Psychology, 74(5), $797-816 . \quad$ doi:10.1037/0022006X.74.5.797

Janoff-Bulman, R. (2006). Schema-change perspectives on posttraumatic growth. In L. Calhoun \& R. Tedeschi (Eds.), Handbook of posttraumatic 
growth: Research and practice (pp. 81-99). Mahwah: Lawrence Erlbaum Associates.

John, P., Russell, S., \& Russell, P. S. (2007). The prevalence of posttraumatic stress disorder among children and adolescents affected by tsunami disaster in Tamil Nadu. Disaster Management \& Response, 5(1), 3-7. doi: 10.1016/j.dmr.2006.11.001

Kilic, C. \& Ulusoy, M. (2003). Psychological effects of the November 1999 earthquake in Turkey: An epidemiological study. Acta Psychiatrica Scandinavica, $\quad 108(3), \quad 232-238$. doi:10.1034/j.1600-0447.2003.00119.x

Kilmer, R. \& Gil- Rivas, V. (2010). Exploring posttraumatic growth in children impacted by Hurricane Katrina: Correlates of the phenomenon and developmental considerations. Child Development, 81(4), 1211-1227. doi:10.1111/j.1467-8624.2010.01463.x

Larrañaga, O. \& Herrera, R. (2011). Encuesta post terremoto: Principales resultados. Efectos en la calidad de vida de la población afectada por el terremoto/tsunami. Santiago, Chile: PNUD/MIDEPLAN. Recuperado de http://www.ministeriodesarrollosocial.gob.cl/enc uesta-post-terremoto/documentos/ informeencuesta-post-terremoto.pdf

Leiva, M. \& Araneda, A, (2013). Prevalencia y sintomatología del estrés post traumático en personas que experimentan un terremoto y un tsunami. Salud y Sociedad, 4(2), 146-151. doi:10.22199/s07187475.2013.0002.00003

McCaughey, R., Hoffman, K. \& Llewellyn, C. (1994). The human experience of earthquake. En R. Ursano, B. McCaughey \& C. Fullerton (Eds.), Individual and community responses to trauma and disaster (pp. 136-153). Cambridge: Cambridge University Press.

McCaslin, S., Zoysa, P., Butler, L., Hart, S., Marmar, C., Metzler, T. \& Koopman, C. (2009). The relationship of posttraumatic growth to peritraumatic reactions and posttraumatic stress symptoms among Sri Lankan University students. Journal of Traumatic Stress, 22(4), 334-339. doi:10.1002/jts.20426

Morris, B., Shakespeare-Finch, J., Rieck, M., \& Newberry, J. (2005). Multidimensional nature of posttraumatic growth in an Australian population. Journal of Traumatic Stress, 18, 575-585. doi:10.1002/jts.20067

Najarian, L., Goenjian, A., Pelcovitz, D., Mandel, F. \& Najarian, B. (2001). The effect of relocation after a natural disaster. Journal of Traumatic Stress, 14(3), 511-526. doi:10.1023/A:1011108622795

Nolen-Hoeksema, S. \& Morrow, J. (1991). A prospective study of depression and posttraumatic stress symptoms after a natural disaster: the 1989 Loma Prieta earthquake. Journal of Personality and Social Psychology, 61, 115-121. doi:10.1037/0022-3514.61.1.115

Olff, M., Langeland, W., Draijer, N. \& Gersons, B. (2007). Gender differences in posttraumatic stress disorder. Psychological Bulletin, 133(2), 183204. doi:10.1037/0033-2909.133.2.183
Orth, U. \& Wieland, E. (2006). Anger, hostility, and posttraumatic stress disorder in trauma-exposed adults: A meta-analysis. Journal of Consulting and Clinical Psychology, 74(4), 698-706. doi:10.1037/0022-006X.74.4.698

Schroevers, M., Helgeson, V., Sanderman, R. \& Ranchor, A. (2010). Type of social support matters for prediction of posttraumatic growth among cancer survivors. Psycho-Oncology, 19, 46-53. doi:10.1002/pon.1501

Shakespeare-Finch, J. \& Lurie-Beck, J. (2013). A metaanalytic clarification of the relationship between posttraumatic growth and symptoms of posttraumatic distress disorder. Journal of Anxiety Disorders, 28(2), 223-229. doi:10.1016/j.janxdis.2013.10.005

Tang, C. S. (2006). Positive and negative postdisaster psychological adjustment among adult survivors of the Southeast Asian earthquake-tsunami. Journal of Psychosomatic Research, 61(5), 699705. doi: 10.1016/j.jpsychores.2006.07.014

Tedeschi, R. \& Calhoun, L. (1996). The Posttraumatic Growth Inventory: Measuring the positive legacy of trauma. Journal of Traumatic Stress, 9(3), 455471. doi: $10.1002 /$ jts. 2490090305

Tedeschi, R. \& Calhoun, L. (2004). Posttraumatic growth: Conceptual foundations and empirical evidence. Psychological Inquiry, 15, 1-18. doi:10.1207/s15327965pli1501_01

Tennen, H. \& Affleck, G. (2005). Benefit-finding and benefitreminding. En C. Snyder \& S. López (Eds.). Handbook of Positive Psychology (pp. 584-597). Oxford: Oxford University Press.

Tolin, D. \& Foa, E. (2006). Sex differences in trauma and posttraumatic stress disorder: A quantitative review of 25 years of research. Psychological Bulletin, 132(6), 959-992. doi:10.1037/00332909.132.6.959

Tomich, P. \& Helgeson, V. (2004). Is finding something good in the bad always good? Benefit finding among women with breast cancer. Health Psychology, 23, 16-23. doi:10.1037/02786133.23.1.16

Trautman, R., Tucker, P., Pfefferbaum, B., Lensgraf, S., Doughty, D., Buksh, A. \& Miller, P. (2002). Effects of prior trauma and age on posttraumatic stress symptoms in Asian and Middle Eastern immigrants after terrorism in the community. Community Mental Health Journal, 38(6), 459474. doi:10.1023/A:1020828117698

Treynor, W., Gonzalez, R. \& Nolen-Hoeksema, S. (2003). Rumination reconsidered: A psychometric analysis. Cognitive Therapy \& Research, 27(3), 247-259. doi:10.1023/A:1023910315561

Tural, U., Aybar Tolun, H., Karakaya, I., Erol, Y., Yildiz, M., \& Erdoğan, S. (2001). Predictors of current comorbid psychiatric disorders with posttraumatic stress disorder in earthquake survivors. Turkish Journal of Psychiatry, 12, 175183.

United States Geological Survey. (2017). Tsunami and earthquake research. Recuperado de 
https://earthquake.usgs.gov/earthquakes/brows e/largest-world.php

Updegraff, J., Taylor, S., Kemeny, M. \& Wyatt, G. (2002). Positive and negative effects of HIV infection in women with low socioeconomic resources. Personality and Social Psychology Bulletin, 28(3), 382-394. doi:10.1177/0146167202286009

Vargas, G., Farías, M., Carretier, S., Tassara, A., Baize, S. \& Melnick, D. (2011). Coastal uplift and tsunami effects associated to the 2010 Mw8. 8 Maule earthquake in Central Chile. Andean Geology, 38(1), 219-238. doi: 10.5027/andgeoV38n1-a12

Vázquez, C., Castilla, C. \& Hervás, G. (2009). Reacciones ante el trauma: Resistencia y crecimiento. En E. Fernández-Abascal (Ed.), Las Emociones Positivas (pp. 375-392). Madrid: Pirámide.

Vishnevsky, T., Cann, A., Calhoun, L., Tedeschi, R. \& Demakis, G. (2010). Gender differences in selfreported posttraumatic growth: A meta-analysis. Psychology of Women Quarterly, 34, 110-120. doi:10.1111/j.1471-6402.2009.01546.x
Weiss, D. \& Marmar, C. (1997). The Impact of Event ScaleRevised. In J. Wilson \& T. Keane (Eds.), Assessing Psychological Trauma and PTSD: A Practitioner's Handbook (pp. 399-411). New York: Guilford Press.

Xu, J. \& Song, X. (2011). Post-traumatic stress disorder among survivors of the Wenchuan earthquake 1 year after: prevalence and risk factors. Comprehensive Psychiatry, 52, 431-437. doi: 10.1016/j.comppsych.2010.08.002

Zoellner, T. \& Maercker, A. (2006a). Post-traumatic growth in clinical psychology. A critical review and introduction of a two component model. Clinical Psychology Review, 26(5), 626-653. doi:10.1016/j.cpr.2006.01.008

Zoellner, T. \& Maercker, A. (2006b). Posttraumatic growth and psychotherapy. In L. Calhoun \& R. Tedeschi (Eds.), Handbook of posttraumatic growth (pp. 334-354). Mahwah: Lawrence Erlbaum Associates. 